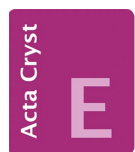

CRYSTALLOGRAPHIC COMMUNICATIONS

ISSN 2056-9890

Received 13 June 2019

Accepted 2 July 2019

Edited by S. Parkin, University of Kentucky, USA

Keywords: crystal structure; 2-(methylamino)tropone; tropolone.

CCDC reference: 1937929

Supporting information: this article has supporting information at journals.iucr.org/e

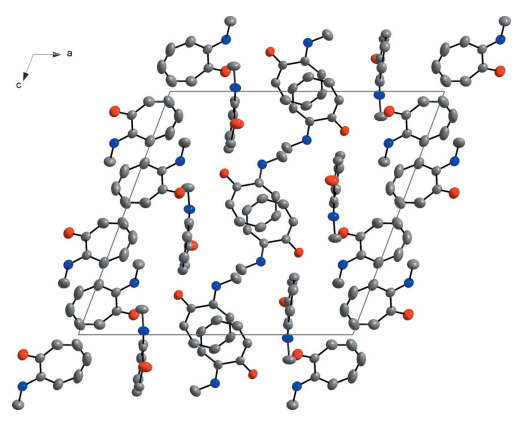

OPEN $\odot$ ACCESS

\section{Crystal structure of 2-(methylamino)tropone}

\author{
Leandri Jansen van Vuuren, Hendrik G. Visser and Marietjie Schutte-Smith*
}

Department of Chemistry, PO Box 339, University of the Free State, Bloemfontein, 9301, South Africa. *Correspondence e-mail: SchutteM@ufs.ac.za

The title compound, 2-(methylamino)cyclohepta-2,4,6-trien-1-one, $\mathrm{C}_{8} \mathrm{H}_{9} \mathrm{NO}$, crystallizes in the monoclinic space group $P 2_{1} / c$, with three independent molecules in the asymmetric unit. The planarity of the molecules is indicated by planes fitted through the seven ring carbon atoms. Small deviations from the planes, with an extremal r.m.s. deviation of $0.0345 \AA$, are present. In complexes of transition metals with similar ligands, the large planar seven-membered aromatic rings have shown to improve the stability of the complex. Two types of hydrogen-bonding interactions, $\mathrm{C}-\mathrm{H} \cdots \mathrm{O}$ and $\mathrm{N}-\mathrm{H} \cdots \mathrm{O}$, are observed, as well as bifurcation of these interactions. The $\mathrm{N}-\mathrm{H} \cdots \mathrm{O}$ interactions link molecules to form infinite chains. The packing of molecules in the unit cell shows a pattern of overlapping aromatic rings, forming column-like formations. $\pi-\pi$ interactions are observed between the overlapping aromatic rings at 3.4462 (19) Å from each other.

\section{Chemical context}

Tropolone and other troponoids, non-benzenoid compounds, have great pharmacological potential (Guo et al., 2019). They display a wide range of bioactivities, including antimicrobial (Saleh et al., 1988), antiviral (Tavis \& Lomonosova, 2015) and antitumor (Ononye et al., 2013) activities. Many tropolonerelated compounds have proved to be possible antiproliferative agents against a variety of cancer cell lines, including lung, prostate and T-cell malignancies (Liu \& Yamauchi, 2006; Hsiao et al., 2012). Tropolone has important medical applications in radiopharmacy (Nepveu et al., 1993) and as catalyst precursor (Crous et al., 2005; Roodt et al., 2003).

Tropolone and its derivatives are versatile ligands used in inorganic and organometallic chemistry (Roesky, 2000; Dias et al., 1995; Nozoe et al., 1997; Schutte et al., 2010; Steyl et al., 2010). The carbonyl oxygen and vicinal coordinating substituent, specifically nitrogen in this study, impart a metalchelating ability to these types of ligands. The complexes of these ligands with first and second row transition elements have increased over the past few decades. The ligands of importance in this study and future work, namely 2-(alkylamino)tropones and aminotroponimines, are $N, O$ and $N, N^{\prime}$ bidentate, monoanionic ligands containing a ten $\pi$-electron backbone (Roesky, 2000). The $\pi$-conjugated backbone is characteristic of these ligands (Nishinaga et al., 2010). Considering the above-mentioned characteristics, tropolone could be considered analogous to the O-donor $\kappa^{2}-O, O^{\prime}$ acetylacetonate ligand (acac- $\left.O, O^{\prime}\right)$. The tropolone bidentate ligand differs from the acac- $O, O^{\prime}$ ligand in a few ways. Of importance to our study is the larger aromatic delocalization, which could afford greater polarizability. Tropolone is also 
more acidic than the acac- $O, O^{\prime}$ ligand. The acidity of the $O, N$ and $N, N^{\prime}$ bidentate ligands used in our study and the effect thereof on the chelating ability could be compared to these $O, O^{\prime}$ bidentate ligands described in the literature. The ligandmetal-ligand angle, better known as the 'bite angle', would also be smaller for a tropolone-derived complex, since it would form a five-membered metallocycle instead of a sixmembered one as with acac- $O, O^{\prime}$ (Bhalla et al., 2005). This could show interesting steric and electronic influences at the metal centre and could be further compared to the steric and electronic studies conveyed on $\beta$-diketone moieties in similar metal complexes by Manicum et al. (2018). These ligands, including the title compound, 2-(methylamino)tropone, will form part of the synthesis of water-soluble complexes of rhenium(I) tricarbonyl, gallium(III) and copper(II). Rhenium(I) (Schutte-Smith et al., 2019), gallium(III) (Green \& Welch, 1989) and copper(II) (Boschi et al., 2018) are highly utilized radioisotopes in the radiopharmaceutical industry.

When designing diagnostic or therapeutic radiopharmaceuticals, certain mechanistic aspects are very important, as it is the basis on which some predictions are made regarding the in vivo behaviour. Kinetic studies, utilizing different techniques, are executed to determine the reaction mechanisms by which the proposed radiopharmaceutical complexes will form and react. Results of such studies are important in nuclear medicine as it gives indications regarding the in vivo stability, uptake and excretion as well as the pharmacokinetics of the compounds. Kinetic investigations by Schutte et al. (2011, 2012), Schutte-Smith et al. (2019) and Manicum et al. (2019) were done on rhenium(I) tricarbonyl tropolonato complexes with satisfying results and conclusions. In the study, methanol substitution was studied using entering nucleophiles in $f a c-\left[\operatorname{Re}(\operatorname{Trop})(\mathrm{CO})_{3}(\mathrm{MeOH})\right]$. The kinetic study performed at high pressure indicated positive volumes of activation for all of the reactions studied. This was a clear indication towards a dissociative interchange mechanism.

The application of these ligands in coordination chemistry could be further increased by adding electron donating or withdrawing moieties to the nitrogen atom.

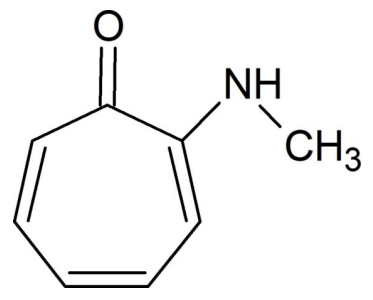

\section{Structural commentary}

2-(Methylamino)tropone crystallizes in the monoclinic $P 2_{1} / c$ space group with three independent molecules, $A, B$ and $C$, in the asymmetric unit (Fig. 1). The bond distances and angles of the three molecules agree well with each other and with those in similar structures (Barret et al., 2014; Dwivedi et al., 2016; Roesky \& Bürgstein, 1999; Shimanouchi \& Sasada, 1973; Siwatch et al., 2011). The largest differences in bond distances are of the $\mathrm{C} 8 B-\mathrm{N} 1 B \quad[1.4470(18) \AA], \quad \mathrm{N} 1 B-\mathrm{C} 2 B$ [1.3444 (17) $\AA]$ and $\mathrm{O} 1 B-\mathrm{C} 1 B[1.2561(15) \AA]$ bonds with the corresponding bonds in 2-(t-butylamino)tropone [1.472 (4) $\AA$; Siwatch et al., 2011], 2-(isopropylamino)tropone [1.330 (4) $\AA$; Roesky \& Bürgstein, 1999] and 2-(t-butylamino)tropone [1.242 (4) §; Siwatch et al., 2011], respectively. Compared to the starting material molecule, tropolone (Shimanouchi \& Sasada, 1973), the O1B-C1B [1.2561 (15) $\AA$ ] bond distance is slightly shorter than that of tropolone [1.261 (3) $\AA$ ], both being in the range of normal $\mathrm{C}=\mathrm{O}$ bond distance. The $\mathrm{C} 2-$ $\mathrm{N} 1-\mathrm{C} 8$ bond angle in molecules $A\left[125.69(13)^{\circ}\right], B$ $\left[125.27(13)^{\circ}\right]$ and $C\left[125.07(12)^{\circ}\right]$ are slightly larger than the usual $120^{\circ}$ for trigonal-planar bond angles, because of the steric influence of the methyl group. These angles are close to the same angle in 2-(benzylamino)tropone [125.09 (12) Barret et al., 2014]. This could be compared to the large angle in 2-(t-butylamino)tropone [131.9 (2) $)^{\circ}$; Siwatch et al., 2011], which deviates even more from $120^{\circ}$ due to the highly steric tertiary butyl group. A plane fitted through the seven ring carbon atoms of the three molecules in the asymmetric unit indicates that the molecules are planar. The root-mean-square deviations of molecules $A, B$ and $C$ from the planes are 0.0141 (12), 0.0261 (11) and 0.0345 (11) $\AA$, respectively. The $\mathrm{C} 8-\mathrm{N} 1-\mathrm{C} 2-\mathrm{C} 3$ torsion angle, which involves the methyl group, differs for molecule $A\left[-0.8(2)^{\circ}\right]$, molecule $B\left[2.3(2)^{\circ}\right]$ and molecule $C\left[7.7(2)^{\circ}\right]$. The small deviations from planarity could possibly be ascribed to the intermolecular hydrogenbonding interactions.

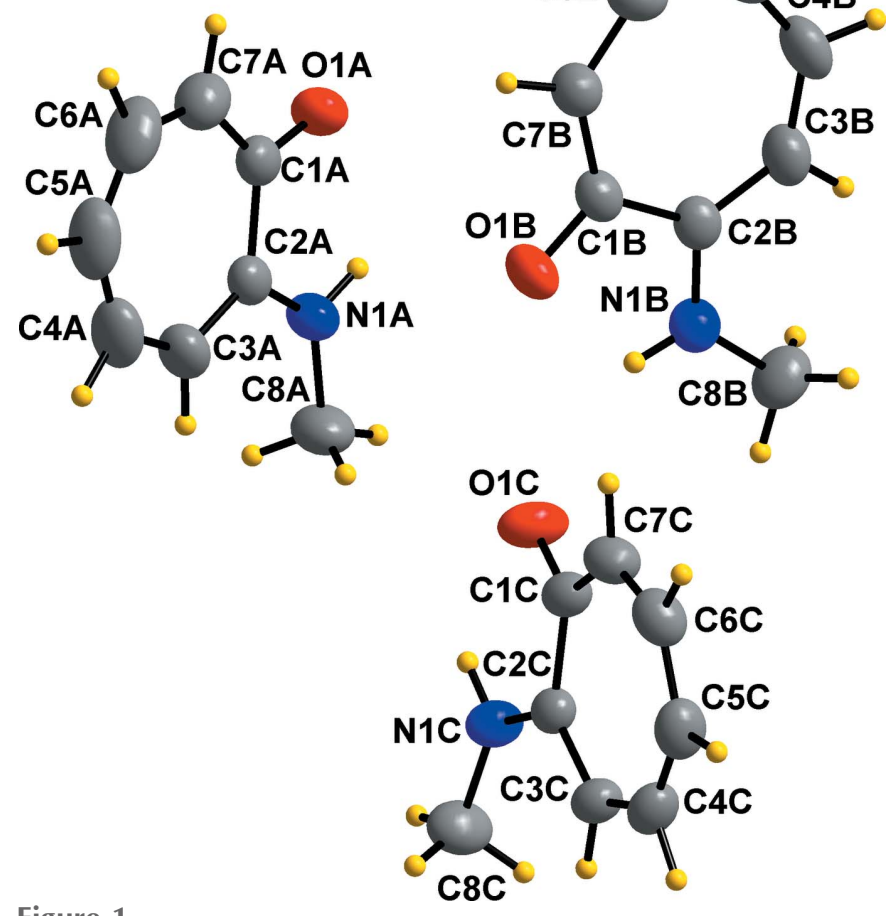

Figure 1

The molecular structure of 2-(methylamino)tropone, indicating the numbering scheme, with displacement ellipsoids drawn at the $50 \%$ probability level. 
Table 1

Hydrogen-bond geometry $\left(\AA,^{\circ}\right)$.

\begin{tabular}{lllll}
\hline$D-\mathrm{H} \cdots A$ & $D-\mathrm{H}$ & $\mathrm{H} \cdots A$ & $D \cdots A$ & $D-\mathrm{H} \cdots A$ \\
\hline $\mathrm{N} 1 A-\mathrm{H} N 1 A \cdots \mathrm{O} 1 A$ & $0.884(17)$ & $2.099(16)$ & $2.5453(16)$ & $110.3(13)$ \\
$\mathrm{N} 1 A-\mathrm{H} 1 A \cdots \mathrm{O} 1 B$ & $0.884(17)$ & $2.248(17)$ & $2.9375(17)$ & $134.6(14)$ \\
$\mathrm{N} 1 B-\mathrm{H} N 1 B \cdots \mathrm{O} 1 B$ & $0.893(15)$ & $2.085(15)$ & $2.5513(16)$ & $111.5(12)$ \\
$\mathrm{N} 1 B-\mathrm{H} N 1 B \cdots \mathrm{O} 1 C$ & $0.893(15)$ & $2.385(15)$ & $3.1566(18)$ & $144.7(13)$ \\
$\mathrm{N} 1 C-\mathrm{H} 1 C \cdots \mathrm{O} 1 C$ & $0.890(16)$ & $2.130(15)$ & $2.5775(16)$ & $110.3(12)$ \\
$\mathrm{N} 1 C-\mathrm{H} N 1 C \cdots \mathrm{O} 1 A^{\mathrm{i}}$ & $0.890(16)$ & $2.313(16)$ & $2.9759(17)$ & $131.3(13)$ \\
$\mathrm{C} 5 C-\mathrm{H} 5 C \cdots \mathrm{O} 1 B^{\mathrm{ii}}$ & 0.95 & 2.42 & $3.2914(19)$ & 153 \\
$\mathrm{C} 7 B-\mathrm{H} 7 B \cdots \mathrm{O} 1 A$ & 0.95 & 2.42 & $3.3446(19)$ & 165 \\
$\mathrm{C} 8 C-\mathrm{H} 8 C 2 \cdots \mathrm{O} 1 A^{\mathrm{i}}$ & 0.98 & 2.56 & $3.178(2)$ & 121 \\
\hline
\end{tabular}

Symmetry codes: (i) $x,-y+\frac{3}{2}, z-\frac{1}{2}$; (ii) $x, y-1, z$.

\section{Supramolecular features}

Nine hydrogen-bonding interactions, three $\mathrm{C}-\mathrm{H} \cdots \mathrm{O}$ and six $\mathrm{N}-\mathrm{H} \cdots \mathrm{O}$, are observed (Table 1 and Fig. 2). Infinite chains are formed along [001]. These supramolecular chains are formed through $\mathrm{N}-\mathrm{H} \cdots \mathrm{O}$ interactions linking the molecules together. As in the crystal structure of tropolone (Shimanouchi \& Sasada, 1973), bifurcation of the hydrogen bonds take place. Bifurcation, also known as the over-coordination of a hydrogen bond, creates both inter- and intramolecular branches, which might contribute to the stability of the structures. This is an interesting phenomenon seen in the orientation of water molecules, where the distribution of acceptor hydrogen bonds, terminating at the lone pairs of the oxygen, is higher (Markovitch \& Agmon, 2008). This forms over-coordinated oxygens and could also be seen in this crystal

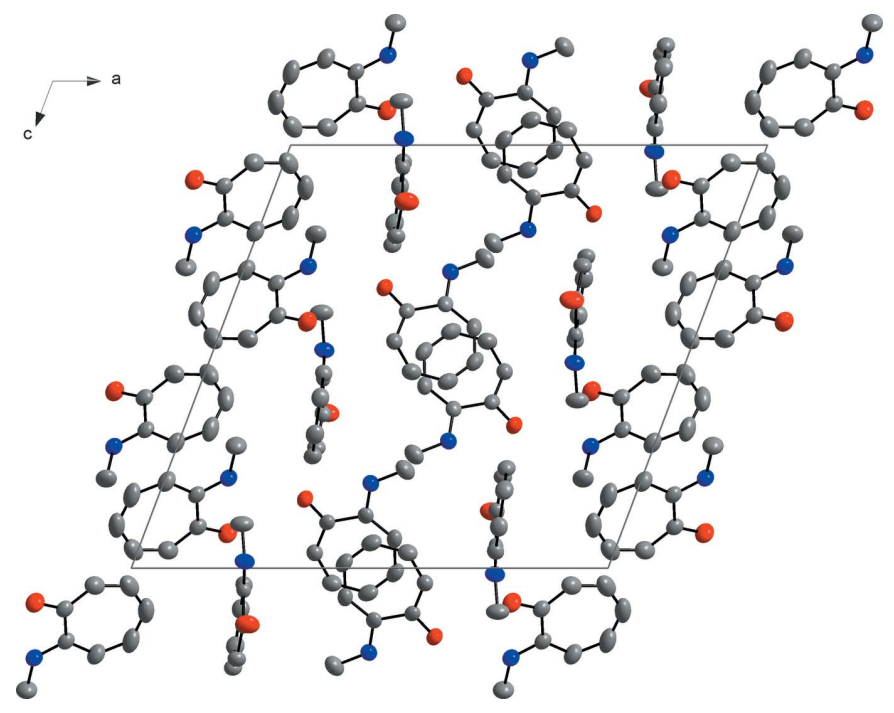

Figure 3

Packing of molecules viewed perpendicular to the $a c$ plane.

structure (Fig. 2). These interactions clearly contribute to the array of the molecules in the asymmetric unit (Fig. 2). The molecules show an interesting packing format in the unit cell. 'Column'-like structures are formed by molecule $B$ packing in a head-to-tail pattern with the aromatic rings overlapping (Fig. 3). A $\pi$-interaction is observed, with a perpendicular distance of 3.4462 (19) $\AA$ between the overlapping aromatic rings of two inversion-related $B$ molecules (Fig. 4). These $\pi$ interactions could not only possibly contribute to the packing

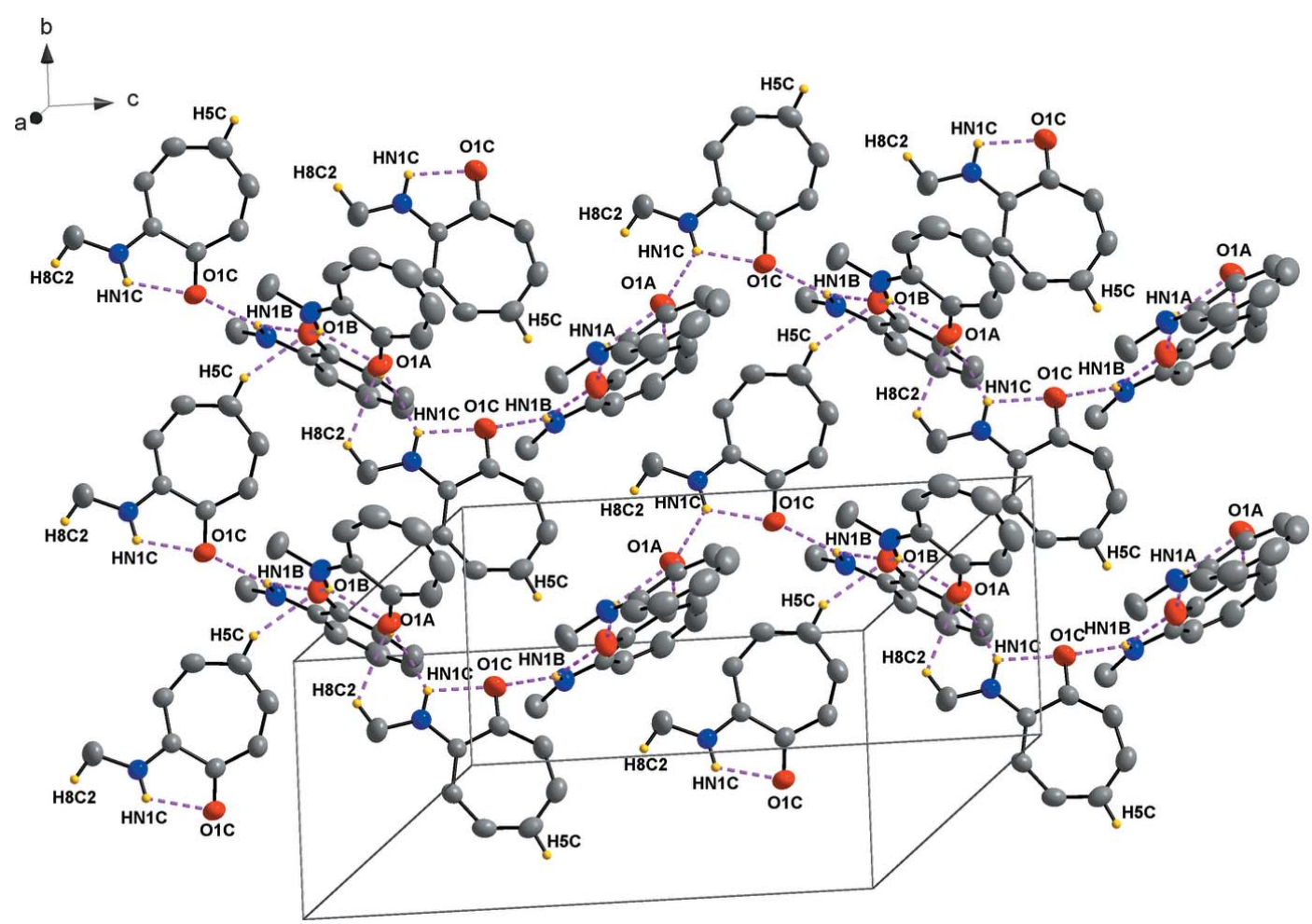

Figure 2

Hydrogen-bonding interactions (Table 1) and infinite chains along [001] in the unit cell. 


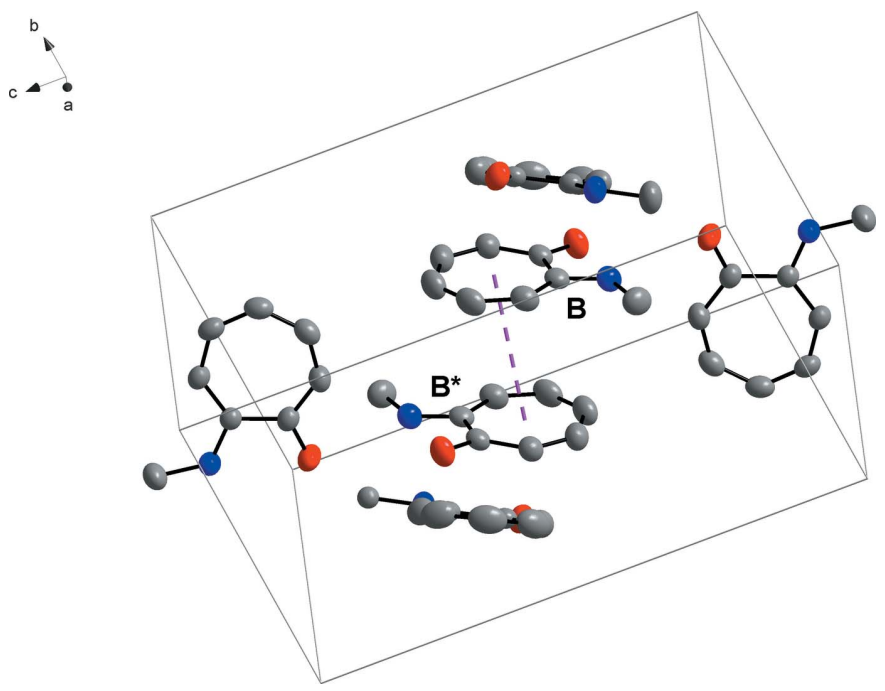

Figure 4

$\pi-\pi$ interaction (highlighted by the dashed line) between overlapping aromatic rings of molecule $B$, where $\mathrm{B}$ and $B^{*}$ are related through inversion.

format of the molecules in the unit cell, but could also assist in the formation of one-dimensional infinite chains, as Wong et al. (2018) have found in water-soluble platinum (II) salts.

\section{Database survey}

A search of the Cambridge Structural Database (CSD, Version 5.40, update of February 2019; Groom et al., 2016) using a $\mathrm{C}_{7} \mathrm{H}_{5} \mathrm{ONH}$ fragment yielded four hits of 2-(alkylamino)tropones. These include 2-(isopropylamino)tropone (LIGVOM: Roesky \& Bürgstein, 1999), 2-(benzylamino)tropone (NOPRUH: Barret et al., 2014), 2-(t-butylamino)tropone (OZINUH: Siwatch et al., 2011) and 2-(cyclo hexylamino)tropone (OTIMUB: Dwivedi et al., 2016). Of the four structures, only the 2-(isopropylamino)tropone and the 2(benzylamino)tropone crystallize in the $P 2_{1} / c$ space group.

\section{Synthesis and crystallization}

Tropolone (505 mg, $4.132 \mathrm{mmol}$ ) was dissolved in $20 \mathrm{~mL}$ of a $40 \%$ methylamine solution. The reaction mixture was stirred at room temperature for $7 \mathrm{~d}$. The product was extracted three times with $30 \mathrm{~mL}$ of chloroform, and the organic layer was washed with $50 \mathrm{~mL}$ of water. The organic layer was dried with $\mathrm{Na}_{2} \mathrm{SO}_{4}$ and the solvent removed under reduced pressure. A $46.03 \%$ yield $(257.1 \mathrm{mg}, 1.902 \mathrm{mmol}$ ) was obtained. Crystals suitable for single crystal X-ray diffraction data collection were obtained by recrystallization from hexane with slow evaporation. Yield: $0.2571 \mathrm{~g}, 46.03 \%$. IR $\left(\mathrm{cm}^{-1}\right): v_{\mathrm{NH}}=3304$, $v_{\mathrm{CO}}=1597$. UV/Vis: $\lambda_{\max }=269 \mathrm{~nm}\left(\varepsilon=1.1885 \times 10^{5}\right.$ $\left.\mathrm{Lmol}^{-1} \mathrm{~cm}^{-1}\right) .{ }^{1} \mathrm{H} \mathrm{NMR}\left(300 \mathrm{MHz}, \mathrm{CDCl}_{3}\right): \delta=7.201(m, 4 \mathrm{H})$, $6.682(t, 1 \mathrm{H}, J=9.6 \mathrm{~Hz}), 6.501(d, 1 \mathrm{H}, J=10.5 \mathrm{~Hz}), 3.056(d$, $3 \mathrm{H}, J=5.4 \mathrm{~Hz}) \cdot{ }^{13} \mathrm{C} \mathrm{NMR}\left(300 \mathrm{MHz}, \mathrm{CDCl}_{3}\right): \delta=177,157,137$, 136, 129, 122, 108, 29.
Table 2

Experimental details.

\begin{tabular}{|c|c|}
\hline \multicolumn{2}{|l|}{ Crystal data } \\
\hline Chemical formula & $\mathrm{C}_{8} \mathrm{H}_{9} \mathrm{NO}$ \\
\hline$M_{\mathrm{r}}$ & 135.16 \\
\hline Crystal system, space group & Monoclinic, $P 2_{1} / c$ \\
\hline Temperature (K) & 100 \\
\hline$a, b, c(\AA)$ & $17.635(5), 7.817(2), 16.718(4)$ \\
\hline$\beta\left(^{\circ}\right)$ & $110.639(9)$ \\
\hline$V\left(\AA^{6}\right)$ & $2156.8(10)$ \\
\hline$Z$ & 12 \\
\hline Radiation type & Мо $K \alpha$ \\
\hline$\mu\left(\mathrm{mm}^{-1}\right)$ & 0.08 \\
\hline Crystal size $(\mathrm{mm})$ & $0.58 \times 0.30 \times 0.28$ \\
\hline \multicolumn{2}{|l|}{ Data collection } \\
\hline Diffractometer & $\begin{array}{l}\text { Bruker X8 APEXII 4K Kappa } \\
\text { CCD }\end{array}$ \\
\hline Absorption correction & $\begin{array}{l}\text { Multi-scan } S A D A B S \text { (Krause } \text { et al., } \\
\text { 2015) }\end{array}$ \\
\hline$T_{\min }, T_{\max }$ & $0.970,0.977$ \\
\hline $\begin{array}{l}\text { No. of measured, independent and } \\
\text { observed }[I>2 \sigma(I)] \text { reflections }\end{array}$ & $33879,5192,3575$ \\
\hline$R_{\text {int }}$ & 0.046 \\
\hline$(\sin \theta / \lambda)_{\max }\left(\AA^{-1}\right)$ & 0.660 \\
\hline \multicolumn{2}{|l|}{ Refinement } \\
\hline$R\left[F^{2}>2 \sigma\left(F^{2}\right)\right], w R\left(F^{2}\right), S$ & $0.039,0.104,1.03$ \\
\hline No. of reflections & 5192 \\
\hline No. of parameters & 287 \\
\hline $\mathrm{H}$-atom treatment & $\begin{array}{l}\mathrm{H} \text { atoms treated by a mixture of } \\
\text { independent and constrained } \\
\text { refinement }\end{array}$ \\
\hline$\Delta \rho_{\max }, \Delta \rho_{\min }\left(\mathrm{e} \AA^{-3}\right)$ & $0.17,-0.13$ \\
\hline
\end{tabular}

Computer programs: APEX2 and SAINT-Plus (Bruker, 2012), SHELXS97 (Sheldrick, 2008), SHELXL2018 (Sheldrick, 2015), DIAMOND (Brandenburg, 2006) and WinGX (Farrugia, 2012).

\section{Refinement}

Crystal data, data collection and structure refinement details are summarized in Table 2. Methyl and aromatic hydrogen atoms were placed in geometrically idealized positions $(\mathrm{C}-\mathrm{H}=0.95-0.98 \AA$ ) and constrained to ride on their parent atoms $\left[U_{\text {iso }}(\mathrm{H})=1.5 U_{\text {eq }}(\mathrm{C})\right.$ and $\left.1.2 U_{\text {eq }}(\mathrm{C})\right]$, while $\mathrm{N}-\mathrm{H}$ hydrogens were freely refined.

\section{Acknowledgements}

This work is based on the research supported in part by the National Research Foundation of South Africa. We would also like to thank the University of the Free State.

\section{References}

Barret, M., Bhatia, P., Kociok-Köhn, G. \& Molloy, K. (2014). Transition Met. Chem. 39, 543-551.

Bhalla, G., Oxgaard, J., Goddard, W. \& Periana, R. (2005). Organometallics, 24, 3229-3232.

Boschi, A., Martini, P., Janevik-Ivanovska, E. \& Duatti, A. (2018). Drug Discovery Today, 23, 1489-1501.

Brandenburg, K. (2006). DIAMOND. Crystal Impact GbR, Bonn, Germany.

Bruker (2012). APEX2, SAINT, Bruker AXS Inc, Madison, Wisconsin, USA.

Crous, R., Datt, M., Foster, D., Bennie, L., Steenkamp, C., Huyser, J., Kirsten, L., Steyl, G. \& Roodt, A. (2005). Dalton Trans. pp. 11081116. 
Dias, H. V. R., Jin, W. \& Ratcliff, R. E. (1995). Inorg. Chem. 34, 61006105.

Dwivedi, A., Binnani, C., Tyagi, D., Rawat, K., Li, P., Zhao, Y., Mobin, S. M., Pathak, B. \& Singh, S. K. (2016). Inorg. Chem. 55, 67396749.

Farrugia, L. J. (2012). J. Appl. Cryst. 45, 849-854.

Green, M. \& Welch, M. (1989). Int. J. Radiat. Appl. Instrum. B, 16, 435-448.

Groom, C. R., Bruno, I. J., Lightfoot, M. P. \& Ward, S. C. (2016). Acta Cryst. B72, 171-179.

Guo, H., Roman, D. \& Beemelmanns, C. (2019). Natural Product Reports. https://doi.org/10.1039/C8NP00078F.

Hsiao, C. J., Hsiao, S. H., Chen, W. L., Guh, J. H., Hsiao, G., Chan, Y. J., Lee, T. H. \& Chung, C. L. (2012). Chem. Biol. Interact. 197, $23-$ 30.

Krause, L., Herbst-Irmer, R., Sheldrick, G. M. \& Stalke, D. (2015). J. Appl. Cryst. 48, 3-10.

Liu, S. \& Yamauchi, H. (2006). Biochem. Biophys. Res. Commun. 351, 26-32.

Manicum, A., Schutte-Smith, M., Alexander, O., Twigge, L., Roodt, A. \& Visser, H. (2019). Inorg. Chem. Commun. 101, 93-98.

Manicum, A., Schutte-Smith, M. \& Visser, H. (2018). Polyhedron, 145, 80-87.

Markovitch, O. \& Agmon, N. (2008). Mol. Phys. 106, 485-495.

Nepveu, F., Jasanada, F. \& Walz, L. (1993). Inorg. Chim. Acta, 211, 141-147.

Nishinaga, T., Aono, T., Isomura, E., Watanabe, S., Miyake, Y., Miyazaki, A., Enoki, T., Miyasaka, H., Otani, H. \& Iyoda, M. (2010). Dalton Trans. 39, 2293-2300.
Nozoe, T., Lin, L. C., Hsu, C., Tsay, S., Hakimelahib, G. H. \& Hwu, J. R. (1997). J. Chem. Res. (S), pp. 362-363.

Ononye, S. N., VanHeyst, M. D., Oblak, E. Z., Zhou, W., Ammar, M., Anderson, A. C. \& Wright, D. L. (2013). ACS Med. Chem. Lett. 4, 757-761.

Roesky, P. \& Bürgstein, M. (1999). Inorg. Chem. 38, 5629-5632.

Roesky, P. W. (2000). Chem. Soc. Rev. 29, 335-345.

Roodt, A., Otto, S. \& Steyl, G. (2003). Coord. Chem. Rev. 245, 121137.

Saleh, N. A., Zfiefak, A., Mordarski, M. \& Pulverer, G. (1988). Zentralbl. Bakteriol. MikroBiol. Hyg. Med. Microbiol. Infect. Dis. Virol. Paras. 270, 160-170.

Schutte, M., Kemp, G., Visser, H. \& Roodt, A. (2011). Inorg. Chem. 50, 12486-12498.

Schutte, M., Roodt, A. \& Visser, H. (2012). Inorg. Chem. 51, 1199612006.

Schutte, M., Visser, H. G. \& Roodt, A. (2010). Acta Cryst. E66, m859$\mathrm{m} 860$.

Schutte-Smith, M., Roodt, A. \& Visser, H. G. (2019). Dalton Trans. https://doi.org/10.1039/C9DT01528K.

Sheldrick, G. M. (2008). Acta Cryst. A64, 112-122.

Sheldrick, G. M. (2015). Acta Cryst. C71, 3-8.

Shimanouchi, H. \& Sasada, Y. (1973). Acta Cryst. B29, 81-90.

Siwatch, R. K., Kundu, S., Kumar, S. \& Nagendran, S. (2011). Organometallics, 30, 1998-2005.

Steyl, G., Muller, T. J. \& Roodt, A. (2010). Acta Cryst. E66, m1508.

Tavis, J. E. \& Lomonosova, E. (2015). Antiviral Res. 118, 132-138.

Wong, V., Po, C., Leung, S., Chan, A., Yang, S., Zhu, B., Cui, X. \& Yam, V. W. (2018). J. Am. Chem. Soc. 140, 657-666. 


\section{supporting information}

Acta Cryst. (2019). E75, 1128-1132［https://doi.org/10.1107/S2056989019009502]

\section{Crystal structure of 2-(methylamino)tropone}

\section{Leandri Jansen van Vuuren, Hendrik G. Visser and Marietjie Schutte-Smith}

Computing details

Data collection: APEX2 (Bruker, 2012); cell refinement: SAINT-Plus (Bruker, 2012); data reduction: SAINT-Plus (Bruker, 2012); program(s) used to solve structure: SHELXS97 (Sheldrick, 2008); program(s) used to refine structure:

SHELXL2018 (Sheldrick, 2015); molecular graphics: DIAMOND (Brandenburg, 2006); software used to prepare material for publication: WinGX (Farrugia, 2012).

2-(Methylamino)cyclohepta-2,4,6-trien-1-one

Crystal data

$\mathrm{C}_{8} \mathrm{H}_{9} \mathrm{NO}$

$M_{r}=135.16$

Monoclinic, $P 2_{1} / c$

Hall symbol: -P 2ybc

$a=17.635(5) \AA$

$b=7.817(2) \AA$

$c=16.718(4) \AA$

$\beta=110.639(9)^{\circ}$

$V=2156.8(10) \AA^{3}$

$Z=12$

$F(000)=864$

$D_{\mathrm{x}}=1.249 \mathrm{Mg} \mathrm{m}^{-3}$

Mo $K \alpha$ radiation, $\lambda=0.71073 \AA$

Cell parameters from 6617 reflections

$\theta=3.5-23.7^{\circ}$

$\mu=0.08 \mathrm{~mm}^{-1}$

$T=100 \mathrm{~K}$

Cuboid, yellow

$0.58 \times 0.30 \times 0.28 \mathrm{~mm}$

\section{Data collection}

Bruker X8 APEXII 4K Kappa CCD diffractometer

Radiation source: fine-focus sealed tube Graphite monochromator $\omega$ scans

Absorption correction: multi-scan

SADABS (Krause et al., 2015)

$T_{\min }=0.970, T_{\max }=0.977$

33879 measured reflections

5192 independent reflections

3575 reflections with $I>2 \sigma(I)$

$R_{\text {int }}=0.046$

$\theta_{\text {max }}=28.0^{\circ}, \theta_{\min }=3.7^{\circ}$

$h=-23 \rightarrow 23$

$k=-10 \rightarrow 10$

$l=-20 \rightarrow 22$

\section{Refinement}

Refinement on $F^{2}$

Least-squares matrix: full

$R\left[F^{2}>2 \sigma\left(F^{2}\right)\right]=0.039$

$w R\left(F^{2}\right)=0.104$

$S=1.03$

5192 reflections

287 parameters

0 restraints

0 constraints

Primary atom site location: structure-invariant direct methods

Secondary atom site location: structureinvariant direct methods

Hydrogen site location: mixed

$\mathrm{H}$ atoms treated by a mixture of independent and constrained refinement

$w=1 /\left[\sigma^{2}\left(F_{0}^{2}\right)+(0.0361 P)^{2}+0.437 P\right]$

where $P=\left(F_{\mathrm{o}}^{2}+2 F_{\mathrm{c}}^{2}\right) / 3$

$(\Delta / \sigma)_{\max }<0.001$

$\Delta \rho_{\max }=0.17 \mathrm{e} \AA^{-3}$

$\Delta \rho_{\min }=-0.13$ e $\AA^{-3}$ 
Extinction correction: SHELXL2018

(Sheldrick, 2015)

Special details

Experimental. The intensity data was collected on a Bruker X8 ApexII 4K Kappa CCD diffractometer using an exposure time of 10 seconds/frame. A total of 1436 frames was collected with a frame width of $0.5^{\circ}$ covering up to $\theta=27.99^{\circ}$ with 99.7\% completeness accomplished.

Geometry. All esds (except the esd in the dihedral angle between two 1.s. planes) are estimated using the full covariance matrix. The cell esds are taken into account individually in the estimation of esds in distances, angles and torsion angles; correlations between esds in cell parameters are only used when they are defined by crystal symmetry. An approximate (isotropic) treatment of cell esds is used for estimating esds involving l.s. planes.

Fractional atomic coordinates and isotropic or equivalent isotropic displacement parameters $\left(\AA^{2}\right)$

\begin{tabular}{|c|c|c|c|c|}
\hline & $x$ & $y$ & $z$ & $U_{\text {iso }} * / U_{\text {eq }}$ \\
\hline $\mathrm{C} 8 \mathrm{~A}$ & $0.12323(10)$ & $0.5412(2)$ & $0.21119(10)$ & $0.0626(4)$ \\
\hline H8A1 & 0.113602 & 0.421239 & 0.221702 & $0.094 *$ \\
\hline H8A2 & 0.172571 & 0.549845 & 0.197169 & $0.094 *$ \\
\hline H8A3 & 0.077051 & 0.584932 & 0.163359 & $0.094^{*}$ \\
\hline $\mathrm{C} 8 \mathrm{~B}$ & $0.49672(10)$ & $0.4930(2)$ & $0.26816(10)$ & $0.0586(4)$ \\
\hline H8B1 & 0.530729 & 0.588791 & 0.263364 & $0.088^{*}$ \\
\hline H8B2 & 0.47058 & 0.440852 & 0.2118 & $0.088^{*}$ \\
\hline H8B3 & 0.530411 & 0.407538 & 0.307747 & $0.088^{*}$ \\
\hline $\mathrm{C} 8 \mathrm{C}$ & $0.19718(11)$ & $0.2656(2)$ & $-0.10383(9)$ & 0.0588 (4) \\
\hline $\mathrm{H} 8 \mathrm{C} 1$ & 0.234672 & 0.189118 & -0.117954 & $0.088^{*}$ \\
\hline $\mathrm{H} 8 \mathrm{C} 2$ & 0.187004 & 0.367098 & -0.140513 & $0.088^{*}$ \\
\hline H8C3 & 0.145975 & 0.20569 & -0.113027 & $0.088^{*}$ \\
\hline N1A & $0.13282(7)$ & $0.64142(16)$ & $0.28726(8)$ & $0.0460(3)$ \\
\hline N1B & $0.43536(8)$ & $0.55487(16)$ & $0.29999(8)$ & $0.0454(3)$ \\
\hline $\mathrm{N} 1 \mathrm{C}$ & $0.23250(7)$ & $0.31715(16)$ & $-0.01509(7)$ & $0.0450(3)$ \\
\hline O1A & $0.17457(6)$ & $0.83578(13)$ & $0.41777(6)$ & $0.0530(3)$ \\
\hline O1B & $0.30974(5)$ & $0.61826(14)$ & $0.33715(6)$ & $0.0515(3)$ \\
\hline $\mathrm{O} 1 \mathrm{C}$ & $0.28810(7)$ & $0.46038(12)$ & $0.13314(6)$ & $0.0574(3)$ \\
\hline HN1A & $0.1803(10)$ & $0.689(2)$ & $0.3149(10)$ & $0.064(5)^{*}$ \\
\hline HN1B & $0.3829(9)$ & $0.5397(19)$ & $0.2695(10)$ & $0.052(4)^{*}$ \\
\hline HN1C & $0.2345(9)$ & $0.428(2)$ & $-0.0016(10)$ & $0.056(5)^{*}$ \\
\hline $\mathrm{C} 1 \mathrm{~B}$ & $0.37461(8)$ & $0.67009(17)$ & $0.39220(8)$ & $0.0379(3)$ \\
\hline $\mathrm{C} 2 \mathrm{~B}$ & $0.44995(7)$ & $0.63079(16)$ & $0.37612(8)$ & $0.0379(3)$ \\
\hline $\mathrm{C} 3 \mathrm{~B}$ & $0.52868(8)$ & $0.66224(19)$ & $0.43132(9)$ & $0.0476(3)$ \\
\hline H3B & 0.569782 & 0.625712 & 0.410663 & $0.057^{*}$ \\
\hline $\mathrm{C} 4 \mathrm{~B}$ & $0.55733(9)$ & $0.7385(2)$ & $0.51194(10)$ & $0.0545(4)$ \\
\hline H4B & 0.614666 & 0.74095 & 0.538203 & $0.065^{*}$ \\
\hline $\mathrm{C} 5 \mathrm{~B}$ & $0.51628(9)$ & $0.8101(2)$ & $0.55919(10)$ & $0.0553(4)$ \\
\hline H5B & 0.548086 & 0.855301 & 0.613404 & $0.066^{*}$ \\
\hline C6B & $0.43262(9)$ & $0.82372(19)$ & $0.53599(9)$ & $0.0510(4)$ \\
\hline H6B & 0.414495 & 0.882838 & 0.575565 & $0.061 *$ \\
\hline C7B & $0.37249(8)$ & $0.76418(18)$ & $0.46432(8)$ & $0.0437(3)$ \\
\hline H7B & 0.319314 & 0.790761 & 0.462424 & $0.052^{*}$ \\
\hline $\mathrm{C} 1 \mathrm{C}$ & $0.27959(8)$ & $0.30140(16)$ & $0.13520(8)$ & $0.0396(3)$ \\
\hline
\end{tabular}




$\begin{array}{lllll}\text { C2C } & 0.25275(7) & 0.21032(16) & 0.05204(8) & 0.0346(3) \\ \text { C3C } & 0.24942(8) & 0.03409(16) & 0.03938(9) & 0.0406(3) \\ \text { H3C } & 0.236584 & -0.000441 & -0.018381 & 0.049^{*} \\ \text { C4C } & 0.26179(8) & -0.09981(17) & 0.09743(9) & 0.0460(3) \\ \text { H4C } & 0.257336 & -0.210933 & 0.073189 & 0.055^{*} \\ \text { C5C } & 0.27941(9) & -0.09661(19) & 0.18414(10) & 0.0495(4) \\ \text { H5C } & 0.283417 & -0.204059 & 0.211879 & 0.059^{*} \\ \text { C6C } & 0.29204(8) & 0.0487(2) & 0.23568(9) & 0.0491(4) \\ \text { H6C } & 0.301651 & 0.026655 & 0.294322 & 0.059^{*} \\ \text { C7C } & 0.29278(8) & 0.21802(19) & 0.21506(9) & 0.0458(3) \\ \text { H7C } & 0.304073 & 0.293672 & 0.262311 & 0.055^{*} \\ \text { C1A } & 0.10733(8) & 0.76484(17) & 0.40289(8) & 0.0411(3) \\ \text { C2A } & 0.07897(8) & 0.65224(16) & 0.32681(8) & 0.0395(3) \\ \text { C3A } & 0.00556(8) & 0.56527(19) & 0.29566(10) & 0.0538(4) \\ \text { H3A } & -0.003564 & 0.505768 & 0.243542 & 0.065^{*} \\ \text { C4A } & -0.05654(9) & 0.5510(2) & 0.32809(13) & 0.0647(5) \\ \text { H4A } & -0.100588 & 0.48099 & 0.295409 & 0.078^{*} \\ \text { C5A } & -0.06451(10) & 0.6213(2) & 0.39924(13) & 0.0702(5) \\ \text { H5A } & -0.112064 & 0.59234 & 0.410595 & 0.084^{*} \\ \text { C6A } & -0.01009(11) & 0.7312(3) & 0.45709(12) & 0.0707(5) \\ \text { H6A } & -0.026039 & 0.768486 & 0.502906 & 0.085^{*} \\ \text { C7A } & 0.06298(10) & 0.7941(2) & 0.45827(10) & 0.0584(4) \\ \text { H7A } & 0.088461 & 0.870606 & 0.504039 & 0.07^{*} \\ & & & & \end{array}$

Atomic displacement parameters $\left(\AA^{2}\right)$

\begin{tabular}{|c|c|c|c|c|c|c|}
\hline & $U^{11}$ & $U^{22}$ & $U^{33}$ & $U^{12}$ & $U^{13}$ & $U^{23}$ \\
\hline $\mathrm{C} 8 \mathrm{~A}$ & $0.0635(10)$ & $0.0735(11)$ & $0.0479(9)$ & $-0.0028(9)$ & $0.0162(8)$ & $-0.0172(8)$ \\
\hline $\mathrm{C} 8 \mathrm{~B}$ & $0.0646(10)$ & $0.0627(10)$ & $0.0585(10)$ & $0.0095(8)$ & $0.0343(8)$ & $0.0034(8)$ \\
\hline $\mathrm{C} 8 \mathrm{C}$ & $0.0758(11)$ & $0.0571(9)$ & $0.0380(8)$ & $0.0003(8)$ & $0.0132(7)$ & $0.0016(7)$ \\
\hline N1A & $0.0412(7)$ & $0.0501(7)$ & $0.0437(7)$ & $-0.0052(6)$ & $0.0114(5)$ & -0.0085 (6) \\
\hline N1B & $0.0432(7)$ & $0.0527(7)$ & $0.0421(7)$ & $0.0019(6)$ & $0.0171(6)$ & $0.0013(5)$ \\
\hline $\mathrm{N} 1 \mathrm{C}$ & $0.0587(7)$ & $0.0373(6)$ & $0.0384(6)$ & $-0.0010(5)$ & $0.0164(5)$ & $-0.0014(5)$ \\
\hline O1A & $0.0484(6)$ & $0.0480(6)$ & $0.0582(6)$ & $-0.0073(5)$ & $0.0132(5)$ & $-0.0137(5)$ \\
\hline O1B & $0.0353(5)$ & $0.0712(7)$ & $0.0441(6)$ & $-0.0060(5)$ & $0.0091(4)$ & $-0.0063(5)$ \\
\hline $\mathrm{O} 1 \mathrm{C}$ & $0.0861(8)$ & $0.0361(5)$ & $0.0494(6)$ & $-0.0083(5)$ & $0.0230(6)$ & $-0.0073(5)$ \\
\hline C1B & $0.0360(7)$ & $0.0408(7)$ & $0.0354(7)$ & $-0.0023(5)$ & $0.0106(5)$ & $0.0074(5)$ \\
\hline $\mathrm{C} 2 \mathrm{~B}$ & $0.0385(7)$ & $0.0371(7)$ & $0.0388(7)$ & $-0.0007(5)$ & $0.0146(6)$ & $0.0094(6)$ \\
\hline C3B & $0.0355(7)$ & $0.0566(9)$ & $0.0517(8)$ & $0.0001(6)$ & $0.0166(6)$ & $0.0091(7)$ \\
\hline C4B & $0.0353(7)$ & $0.0669(10)$ & $0.0522(9)$ & $-0.0082(7)$ & $0.0042(6)$ & $0.0085(8)$ \\
\hline C5B & $0.0516(9)$ & $0.0634(10)$ & $0.0422(8)$ & $-0.0135(7)$ & $0.0056(7)$ & $-0.0022(7)$ \\
\hline C6B & $0.0576(9)$ & $0.0530(9)$ & $0.0425(8)$ & $-0.0058(7)$ & $0.0177(7)$ & $-0.0046(7)$ \\
\hline C7B & $0.0409(7)$ & $0.0497(8)$ & $0.0417(7)$ & $-0.0007(6)$ & $0.0158(6)$ & $0.0021(6)$ \\
\hline $\mathrm{C} 1 \mathrm{C}$ & $0.0402(7)$ & $0.0381(7)$ & $0.0416(7)$ & $-0.0022(5)$ & $0.0158(6)$ & $-0.0058(6)$ \\
\hline $\mathrm{C} 2 \mathrm{C}$ & $0.0327(6)$ & $0.0361(6)$ & $0.0365(7)$ & $0.0003(5)$ & $0.0141(5)$ & -0.0009 (5) \\
\hline $\mathrm{C} 3 \mathrm{C}$ & $0.0445(7)$ & $0.0367(7)$ & $0.0417(7)$ & $-0.0003(6)$ & $0.0164(6)$ & $-0.0054(6)$ \\
\hline $\mathrm{C} 4 \mathrm{C}$ & $0.0478(8)$ & $0.0337(7)$ & $0.0571(9)$ & $0.0026(6)$ & $0.0192(7)$ & $-0.0010(6)$ \\
\hline $\mathrm{C} 5 \mathrm{C}$ & $0.0486(8)$ & $0.0423(8)$ & $0.0561(9)$ & $0.0042(6)$ & $0.0165(7)$ & $0.0120(7)$ \\
\hline
\end{tabular}


supporting information

\begin{tabular}{lllllll} 
C6C & $0.0462(8)$ & $0.0590(9)$ & $0.0393(8)$ & $-0.0020(7)$ & $0.0117(6)$ & $0.0074(7)$ \\
C7C & $0.0492(8)$ & $0.0502(8)$ & $0.0372(7)$ & $-0.0054(6)$ & $0.0143(6)$ & $-0.0050(6)$ \\
C1A & $0.0396(7)$ & $0.0367(7)$ & $0.0420(7)$ & $0.0057(6)$ & $0.0080(6)$ & $0.0033(6)$ \\
C2A & $0.0368(7)$ & $0.0347(7)$ & $0.0417(7)$ & $0.0039(5)$ & $0.0072(6)$ & $0.0032(6)$ \\
C3A & $0.0406(8)$ & $0.0510(8)$ & $0.0620(10)$ & $-0.0038(6)$ & $0.0086(7)$ & $-0.0062(7)$ \\
C4A & $0.0392(8)$ & $0.0596(10)$ & $0.0907(13)$ & $-0.0038(7)$ & $0.0170(8)$ & $0.0064(9)$ \\
C5A & $0.0445(9)$ & $0.0794(12)$ & $0.0920(14)$ & $0.0088(9)$ & $0.0308(9)$ & $0.0247(11)$ \\
C6A & $0.0661(11)$ & $0.0884(13)$ & $0.0683(11)$ & $0.0241(10)$ & $0.0372(10)$ & $0.0169(10)$ \\
C7A & $0.0603(10)$ & $0.0643(10)$ & $0.0504(9)$ & $0.0113(8)$ & $0.0194(8)$ & $-0.0021(8)$ \\
\hline
\end{tabular}

Geometric parameters $\left(\AA,{ }^{\circ}\right)$

\begin{tabular}{|c|c|c|c|}
\hline $\mathrm{C} 8 \mathrm{~A}-\mathrm{N} 1 \mathrm{~A}$ & $1.4518(19)$ & $\mathrm{C} 4 \mathrm{~B}-\mathrm{H} 4 \mathrm{~B}$ & 0.95 \\
\hline $\mathrm{C} 8 \mathrm{~A}-\mathrm{H} 8 \mathrm{~A} 1$ & 0.98 & $\mathrm{C} 5 \mathrm{~B}-\mathrm{C} 6 \mathrm{~B}$ & $1.391(2)$ \\
\hline $\mathrm{C} 8 \mathrm{~A}-\mathrm{H} 8 \mathrm{~A} 2$ & 0.98 & $\mathrm{C} 5 \mathrm{~B}-\mathrm{H} 5 \mathrm{~B}$ & 0.95 \\
\hline $\mathrm{C} 8 \mathrm{~A}-\mathrm{H} 8 \mathrm{~A} 3$ & 0.98 & $\mathrm{C} 6 \mathrm{~B}-\mathrm{C} 7 \mathrm{~B}$ & $1.3716(19)$ \\
\hline $\mathrm{C} 8 \mathrm{~B}-\mathrm{N} 1 \mathrm{~B}$ & $1.4470(18)$ & C6B-H6B & 0.95 \\
\hline C8B-H8B1 & 0.98 & $\mathrm{C} 7 \mathrm{~B}-\mathrm{H} 7 \mathrm{~B}$ & 0.95 \\
\hline $\mathrm{C} 8 \mathrm{~B}-\mathrm{H} 8 \mathrm{~B} 2$ & 0.98 & $\mathrm{C} 1 \mathrm{C}-\mathrm{C} 7 \mathrm{C}$ & $1.4293(19)$ \\
\hline $\mathrm{C} 8 \mathrm{~B}-\mathrm{H} 8 \mathrm{~B} 3$ & 0.98 & $\mathrm{C} 1 \mathrm{C}-\mathrm{C} 2 \mathrm{C}$ & $1.4832(18)$ \\
\hline $\mathrm{C} 8 \mathrm{C}-\mathrm{N} 1 \mathrm{C}$ & $1.4492(18)$ & $\mathrm{C} 2 \mathrm{C}-\mathrm{C} 3 \mathrm{C}$ & $1.3919(18)$ \\
\hline $\mathrm{C} 8 \mathrm{C}-\mathrm{H} 8 \mathrm{C} 1$ & 0.98 & $\mathrm{C} 3 \mathrm{C}-\mathrm{C} 4 \mathrm{C}$ & $1.3913(19)$ \\
\hline $\mathrm{C} 8 \mathrm{C}-\mathrm{H} 8 \mathrm{C} 2$ & 0.98 & $\mathrm{C} 3 \mathrm{C}-\mathrm{H} 3 \mathrm{C}$ & 0.95 \\
\hline $\mathrm{C} 8 \mathrm{C}-\mathrm{H} 8 \mathrm{C} 3$ & 0.98 & $\mathrm{C} 4 \mathrm{C}-\mathrm{C} 5 \mathrm{C}$ & $1.372(2)$ \\
\hline $\mathrm{N} 1 \mathrm{~A}-\mathrm{C} 2 \mathrm{~A}$ & $1.3376(18)$ & $\mathrm{C} 4 \mathrm{C}-\mathrm{H} 4 \mathrm{C}$ & 0.95 \\
\hline N1A-HN1A & $0.884(17)$ & $\mathrm{C} 5 \mathrm{C}-\mathrm{C} 6 \mathrm{C}$ & $1.395(2)$ \\
\hline $\mathrm{N} 1 \mathrm{~B}-\mathrm{C} 2 \mathrm{~B}$ & $1.3444(17)$ & $\mathrm{C} 5 \mathrm{C}-\mathrm{H} 5 \mathrm{C}$ & 0.95 \\
\hline N1B-HN1B & $0.893(15)$ & $\mathrm{C} 6 \mathrm{C}-\mathrm{C} 7 \mathrm{C}$ & $1.369(2)$ \\
\hline $\mathrm{N} 1 \mathrm{C}-\mathrm{C} 2 \mathrm{C}$ & $1.3423(16)$ & $\mathrm{C} 6 \mathrm{C}-\mathrm{H} 6 \mathrm{C}$ & 0.95 \\
\hline $\mathrm{N} 1 \mathrm{C}-\mathrm{HN} 1 \mathrm{C}$ & $0.890(16)$ & $\mathrm{C} 7 \mathrm{C}-\mathrm{H} 7 \mathrm{C}$ & 0.95 \\
\hline $\mathrm{O} 1 \mathrm{~A}-\mathrm{O} 1 \mathrm{~A}$ & $0.000(3)$ & $\mathrm{C} 1 \mathrm{~A}-\mathrm{C} 7 \mathrm{~A}$ & $1.426(2)$ \\
\hline $\mathrm{O} 1 \mathrm{~A}-\mathrm{C} 1 \mathrm{~A}$ & $1.2519(16)$ & $\mathrm{C} 1 \mathrm{~A}-\mathrm{C} 2 \mathrm{~A}$ & $1.4812(19)$ \\
\hline $\mathrm{O} 1 \mathrm{~B}-\mathrm{O} 1 \mathrm{~B}$ & $0.0000(19)$ & $\mathrm{C} 2 \mathrm{~A}-\mathrm{C} 3 \mathrm{~A}$ & $1.3906(19)$ \\
\hline $\mathrm{O} 1 \mathrm{~B}-\mathrm{C} 1 \mathrm{~B}$ & $1.2561(15)$ & $\mathrm{C} 3 \mathrm{~A}-\mathrm{C} 4 \mathrm{~A}$ & $1.387(2)$ \\
\hline $\mathrm{O} 1 \mathrm{C}-\mathrm{O} 1 \mathrm{C}$ & $0.000(3)$ & $\mathrm{C} 3 \mathrm{~A}-\mathrm{H} 3 \mathrm{~A}$ & 0.95 \\
\hline $\mathrm{O} 1 \mathrm{C}-\mathrm{C} 1 \mathrm{C}$ & $1.2537(16)$ & $\mathrm{C} 4 \mathrm{~A}-\mathrm{C} 5 \mathrm{~A}$ & $1.362(3)$ \\
\hline $\mathrm{C} 1 \mathrm{~B}-\mathrm{C} 7 \mathrm{~B}$ & $1.4240(19)$ & $\mathrm{C} 4 \mathrm{~A}-\mathrm{H} 4 \mathrm{~A}$ & 0.95 \\
\hline $\mathrm{C} 1 \mathrm{~B}-\mathrm{C} 2 \mathrm{~B}$ & $1.4777(18)$ & $\mathrm{C} 5 \mathrm{~A}-\mathrm{C} 6 \mathrm{~A}$ & $1.393(3)$ \\
\hline $\mathrm{C} 2 \mathrm{~B}-\mathrm{C} 3 \mathrm{~B}$ & $1.3917(19)$ & $\mathrm{C} 5 \mathrm{~A}-\mathrm{H} 5 \mathrm{~A}$ & 0.95 \\
\hline $\mathrm{C} 3 \mathrm{~B}-\mathrm{C} 4 \mathrm{~B}$ & $1.395(2)$ & $\mathrm{C} 6 \mathrm{~A}-\mathrm{C} 7 \mathrm{~A}$ & $1.373(2)$ \\
\hline $\mathrm{C} 3 \mathrm{~B}-\mathrm{H} 3 \mathrm{~B}$ & 0.95 & $\mathrm{C} 6 \mathrm{~A}-\mathrm{H} 6 \mathrm{~A}$ & 0.95 \\
\hline $\mathrm{C} 4 \mathrm{~B}-\mathrm{C} 5 \mathrm{~B}$ & $1.366(2)$ & $\mathrm{C} 7 \mathrm{~A}-\mathrm{H} 7 \mathrm{~A}$ & 0.95 \\
\hline $\mathrm{N} 1 \mathrm{~A}-\mathrm{C} 8 \mathrm{~A}-\mathrm{H} 8 \mathrm{~A} 1$ & 109.5 & $\mathrm{C} 6 \mathrm{~B}-\mathrm{C} 7 \mathrm{~B}-\mathrm{C} 1 \mathrm{~B}$ & $132.22(14)$ \\
\hline $\mathrm{N} 1 \mathrm{~A}-\mathrm{C} 8 \mathrm{~A}-\mathrm{H} 8 \mathrm{~A} 2$ & 109.5 & $\mathrm{C} 6 \mathrm{~B}-\mathrm{C} 7 \mathrm{~B}-\mathrm{H} 7 \mathrm{~B}$ & 113.9 \\
\hline $\mathrm{H} 8 \mathrm{~A} 1-\mathrm{C} 8 \mathrm{~A}-\mathrm{H} 8 \mathrm{~A} 2$ & 109.5 & $\mathrm{C} 1 \mathrm{~B}-\mathrm{C} 7 \mathrm{~B}-\mathrm{H} 7 \mathrm{~B}$ & 113.9 \\
\hline $\mathrm{N} 1 \mathrm{~A}-\mathrm{C} 8 \mathrm{~A}-\mathrm{H} 8 \mathrm{~A} 3$ & 109.5 & $\mathrm{O} 1 \mathrm{C}-\mathrm{C} 1 \mathrm{C}-\mathrm{O} 1 \mathrm{C}$ & $0.00(10)$ \\
\hline $\mathrm{H} 8 \mathrm{~A} 1-\mathrm{C} 8 \mathrm{~A}-\mathrm{H} 8 \mathrm{~A} 3$ & 109.5 & $\mathrm{O} 1 \mathrm{C}-\mathrm{C} 1 \mathrm{C}-\mathrm{C} 7 \mathrm{C}$ & $119.73(12)$ \\
\hline
\end{tabular}




$\begin{array}{ll}\text { H8A2-C8A-H8A3 } & 109.5 \\ \text { N1B-C8B-H8B1 } & 109.5 \\ \text { N1B-C8B-H8B2 } & 109.5 \\ \text { H8B1-C8B-H8B2 } & 109.5 \\ \text { N1B-C8B-H8B3 } & 109.5 \\ \text { H8B1-C8B-H8B3 } & 109.5 \\ \text { H8B2-C8B-H8B3 } & 109.5 \\ \text { N1C-C8C-H8C1 } & 109.5 \\ \text { N1C-C8C-H8C2 } & 109.5 \\ \text { H8C1-C8C-H8C2 } & 109.5 \\ \text { N1C-C8C-H8C3 } & 109.5 \\ \text { H8C1-C8C-H8C3 } & 109.5 \\ \text { H8C2-C8C-H8C3 } & 109.5 \\ \text { C2A-N1A-C8A } & 125.69(13) \\ \text { C2A-N1A-HN1A } & 115.0(11) \\ \text { C8A-N1A-HN1A } & 118.8(11) \\ \text { C2B-N1B-C8B } & 125.27(13) \\ \text { C2B-N1B-HN1B } & 114.4(10) \\ \text { C8B-N1B-HN1B } & 120.3(10) \\ \text { C2C-N1C-C8C } & 125.07(12) \\ \text { C2C-N1C-HN1C } & 114.7(10) \\ \text { C8C-N1C-HN1C } & 119.5(10) \\ \text { O1A-O1A-C1A } & 0(10) \\ \text { O1B-O1B-C1B } & 0(10) \\ \text { O1C-O1C-C1C } & 0(10) \\ \text { O1B-C1B-O1B } & 0.00(15) \\ \text { O1B-C1B-C7B } & 119.87(12) \\ \text { O1B-C1B-C7B } & 119.87(12) \\ \text { O1B-C1B-C2B } & 116.40(12) \\ \text { O1B-C1B-C2B } & 116.40(12) \\ \text { C7B-C1B-C2B } & 123.72(12) \\ \text { N1B-C2B-C3B } & 121.26(12) \\ \text { N1B-C2B-C1B } & 112.32(11) \\ \text { C3B-C2B-C1B } & 126.40(13) \\ \text { C2B-C3B-C4B } & 130.76(14) \\ \text { C2B-C3B-H3B } & 114.6 \\ \text { C4B-C3B-H3B } & 114.6 \\ \text { C5B-C4B-C3B } & 130.44(14) \\ \text { C5B-C4B-H4B } & 114.8 \\ \text { C3B-C4B-H4B } & 114.8 \\ \text { C4B-C5B-C6B } & 126.62(14) \\ \text { C4B-C5B-H5B } & 116.7 \\ \text { C6B-C5B-H5B } & 116.7 \\ \text { C7B-C6B-C5B } & 129.43(15) \\ \text { C7B-C6B-H6B } & 115.3 \\ \text { C5B-C6B-H6B } & \\ & \\ \text { O1B-C1B-C7B } & \\ & \end{array}$

$\mathrm{O} 1 \mathrm{C}-\mathrm{C} 1 \mathrm{C}-\mathrm{C} 7 \mathrm{C}$

$\mathrm{O} 1 \mathrm{C}-\mathrm{C} 1 \mathrm{C}-\mathrm{C} 2 \mathrm{C}$

$\mathrm{O} 1 \mathrm{C}-\mathrm{C} 1 \mathrm{C}-\mathrm{C} 2 \mathrm{C}$

$\mathrm{C} 7 \mathrm{C}-\mathrm{C} 1 \mathrm{C}-\mathrm{C} 2 \mathrm{C}$

$\mathrm{N} 1 \mathrm{C}-\mathrm{C} 2 \mathrm{C}-\mathrm{C} 3 \mathrm{C}$

$\mathrm{N} 1 \mathrm{C}-\mathrm{C} 2 \mathrm{C}-\mathrm{C} 1 \mathrm{C}$

$\mathrm{C} 3 \mathrm{C}-\mathrm{C} 2 \mathrm{C}-\mathrm{C} 1 \mathrm{C}$

$\mathrm{C} 4 \mathrm{C}-\mathrm{C} 3 \mathrm{C}-\mathrm{C} 2 \mathrm{C}$

$\mathrm{C} 4 \mathrm{C}-\mathrm{C} 3 \mathrm{C}-\mathrm{H} 3 \mathrm{C}$

$\mathrm{C} 2 \mathrm{C}-\mathrm{C} 3 \mathrm{C}-\mathrm{H} 3 \mathrm{C}$

$\mathrm{C} 5 \mathrm{C}-\mathrm{C} 4 \mathrm{C}-\mathrm{C} 3 \mathrm{C}$

$\mathrm{C} 5 \mathrm{C}-\mathrm{C} 4 \mathrm{C}-\mathrm{H} 4 \mathrm{C}$

$\mathrm{C} 3 \mathrm{C}-\mathrm{C} 4 \mathrm{C}-\mathrm{H} 4 \mathrm{C}$

$\mathrm{C} 4 \mathrm{C}-\mathrm{C} 5 \mathrm{C}-\mathrm{C} 6 \mathrm{C}$

$\mathrm{C} 4 \mathrm{C}-\mathrm{C} 5 \mathrm{C}-\mathrm{H} 5 \mathrm{C}$

$\mathrm{C} 6 \mathrm{C}-\mathrm{C} 5 \mathrm{C}-\mathrm{H} 5 \mathrm{C}$

$\mathrm{C} 7 \mathrm{C}-\mathrm{C} 6 \mathrm{C}-\mathrm{C} 5 \mathrm{C}$

$\mathrm{C} 7 \mathrm{C}-\mathrm{C} 6 \mathrm{C}-\mathrm{H} 6 \mathrm{C}$

$\mathrm{C} 5 \mathrm{C}-\mathrm{C} 6 \mathrm{C}-\mathrm{H} 6 \mathrm{C}$

$\mathrm{C} 6 \mathrm{C}-\mathrm{C} 7 \mathrm{C}-\mathrm{C} 1 \mathrm{C}$

$\mathrm{C} 6 \mathrm{C}-\mathrm{C} 7 \mathrm{C}-\mathrm{H} 7 \mathrm{C}$

$\mathrm{C} 1 \mathrm{C}-\mathrm{C} 7 \mathrm{C}-\mathrm{H} 7 \mathrm{C}$

$\mathrm{O} 1 \mathrm{~A}-\mathrm{C} 1 \mathrm{~A}-\mathrm{O} 1 \mathrm{~A}$

O1A-C1A-C7A

$\mathrm{O} 1 \mathrm{~A}-\mathrm{C} 1 \mathrm{~A}-\mathrm{C} 7 \mathrm{~A}$

$\mathrm{O} 1 \mathrm{~A}-\mathrm{C} 1 \mathrm{~A}-\mathrm{C} 2 \mathrm{~A}$

$\mathrm{O} 1 \mathrm{~A}-\mathrm{C} 1 \mathrm{~A}-\mathrm{C} 2 \mathrm{~A}$

$\mathrm{C} 7 \mathrm{~A}-\mathrm{C} 1 \mathrm{~A}-\mathrm{C} 2 \mathrm{~A}$

$\mathrm{N} 1 \mathrm{~A}-\mathrm{C} 2 \mathrm{~A}-\mathrm{C} 3 \mathrm{~A}$

$\mathrm{N} 1 \mathrm{~A}-\mathrm{C} 2 \mathrm{~A}-\mathrm{C} 1 \mathrm{~A}$

$\mathrm{C} 3 \mathrm{~A}-\mathrm{C} 2 \mathrm{~A}-\mathrm{C} 1 \mathrm{~A}$

$\mathrm{C} 4 \mathrm{~A}-\mathrm{C} 3 \mathrm{~A}-\mathrm{C} 2 \mathrm{~A}$

$\mathrm{C} 4 \mathrm{~A}-\mathrm{C} 3 \mathrm{~A}-\mathrm{H} 3 \mathrm{~A}$

$\mathrm{C} 2 \mathrm{~A}-\mathrm{C} 3 \mathrm{~A}-\mathrm{H} 3 \mathrm{~A}$

$\mathrm{C} 5 \mathrm{~A}-\mathrm{C} 4 \mathrm{~A}-\mathrm{C} 3 \mathrm{~A}$

$\mathrm{C} 5 \mathrm{~A}-\mathrm{C} 4 \mathrm{~A}-\mathrm{H} 4 \mathrm{~A}$

$\mathrm{C} 3 \mathrm{~A}-\mathrm{C} 4 \mathrm{~A}-\mathrm{H} 4 \mathrm{~A}$

$\mathrm{C} 4 \mathrm{~A}-\mathrm{C} 5 \mathrm{~A}-\mathrm{C} 6 \mathrm{~A}$

$\mathrm{C} 4 \mathrm{~A}-\mathrm{C} 5 \mathrm{~A}-\mathrm{H} 5 \mathrm{~A}$

$\mathrm{C} 6 \mathrm{~A}-\mathrm{C} 5 \mathrm{~A}-\mathrm{H} 5 \mathrm{~A}$

$\mathrm{C} 7 \mathrm{~A}-\mathrm{C} 6 \mathrm{~A}-\mathrm{C} 5 \mathrm{~A}$

C7A-C6A-H6A

C5A-C6A-H6A

$\mathrm{C} 6 \mathrm{~A}-\mathrm{C} 7 \mathrm{~A}-\mathrm{C} 1 \mathrm{~A}$

C6A-C7A-H7A

$\mathrm{C} 1 \mathrm{~A}-\mathrm{C} 7 \mathrm{~A}-\mathrm{H} 7 \mathrm{~A}$

$\mathrm{N} 1 \mathrm{C}-\mathrm{C} 2 \mathrm{C}-\mathrm{C} 3 \mathrm{C}-\mathrm{C} 4 \mathrm{C}$
$119.73(12)$

$116.86(12)$

$116.86(12)$

$123.36(12)$

$120.28(12)$

$112.83(11)$

$126.87(12)$

$130.60(13)$

114.7

114.7

$130.16(13)$

114.9

114.9

$126.51(13)$

116.7

116.7

$130.18(14)$

114.9

114.9

$131.55(13)$

114.2

114.2

$0.00(9)$

$119.86(13)$

$119.86(13)$

$116.32(12)$

$116.32(12)$

123.82 (13)

120.88 (13)

112.23 (11)

$126.88(13)$

130.67 (16)

114.7

114.7

130.39 (16)

114.8

114.8

126.67 (16)

116.7

116.7

130.12 (17)

114.9

114.9

$131.32(16)$

114.3

114.3

$-174.98(13)$ 


$\begin{array}{ll}\text { O1B-O1B-C1B-C2B } & 0.00(11) \\ \text { C8B-N1B-C2B-C3B } & 2.3(2) \\ \text { C8B-N1B-C2B-C1B } & -176.57(13) \\ \text { O1B-C1B-C2B-N1B } & 4.66(16) \\ \text { O1B-C1B-C2B-N1B } & 4.66(16) \\ \text { C7B-C1B-C2B-N1B } & -174.08(12) \\ \text { O1B-C1B-C2B-C3B } & -174.13(13) \\ \text { O1B-C1B-C2B-C3B } & -174.13(13) \\ \text { C7B-C1B-C2B-C3B } & 7.1(2) \\ \text { N1B-C2B-C3B-C4B } & 179.84(15) \\ \text { C1B-C2B-C3B-C4B } & -1.5(2) \\ \text { C2B-C3B-C4B-C5B } & -2.6(3) \\ \text { C3B-C4B-C5B-C6B } & -0.1(3) \\ \text { C4B-C5B-C6B-C7B } & 3.1(3) \\ \text { C5B-C6B-C7B-C1B } & 0.8(3) \\ \text { O1B-C1B-C7B-C6B } & 174.25(15) \\ \text { O1B-C1B-C7B-C6B } & 174.25(15) \\ \text { C2B-C1B-C7B-C6B } & -7.1(2) \\ \text { O1C-O1C-C1C-C7C } & 0.0(2) \\ \text { O1C-O1C-C1C-C2C } & 0.0(2) \\ \text { C8C-N1C-C2C-C3C } & 7.7(2) \\ \text { C8C-N1C-C2C-C1C } & -174.00(13) \\ \text { O1C-C1C-C2C-N1C } & -7.16(17) \\ \text { O1C-C1C-C2C-N1C } & -7.16(17) \\ \text { C7C-C1C-C2C-N1C } & 170.43(12) \\ \text { O1C-C1C-C2C-C3C } & 171.01(13) \\ \text { O1C-C1C-C2C-C3C } & 171.01(13) \\ \text { C7C-C1C-C2C-C3C } & -11.4(2) \\ \end{array}$

$\begin{array}{ll}\mathrm{C} 1 \mathrm{C}-\mathrm{C} 2 \mathrm{C}-\mathrm{C} 3 \mathrm{C}-\mathrm{C} 4 \mathrm{C} & 7.0(2) \\ \mathrm{C} 2 \mathrm{C}-\mathrm{C} 3 \mathrm{C}-\mathrm{C} 4 \mathrm{C}-\mathrm{C} 5 \mathrm{C} & 1.4(3) \\ \mathrm{C} 3 \mathrm{C}-\mathrm{C} 4 \mathrm{C}-\mathrm{C} 5 \mathrm{C}-\mathrm{C} 6 \mathrm{C} & -2.5(3) \\ \mathrm{C} 4 \mathrm{C}-\mathrm{C} 5 \mathrm{C}-\mathrm{C} 6 \mathrm{C}-\mathrm{C} 7 \mathrm{C} & -2.3(3) \\ \mathrm{C} 5 \mathrm{C}-\mathrm{C} 6 \mathrm{C}-\mathrm{C} 7 \mathrm{C}-\mathrm{C} 1 \mathrm{C} & 1.4(3) \\ \mathrm{O} 1 \mathrm{C}-\mathrm{C} 1 \mathrm{C}-\mathrm{C} 7 \mathrm{C}-\mathrm{C} 6 \mathrm{C} & -175.92(15) \\ \mathrm{O} 1 \mathrm{C}-\mathrm{C} 1 \mathrm{C}-\mathrm{C} 7 \mathrm{C}-\mathrm{C} 6 \mathrm{C} & -175.92(15) \\ \mathrm{C} 2 \mathrm{C}-\mathrm{C} 1 \mathrm{C}-\mathrm{C} 7 \mathrm{C}-\mathrm{C} 6 \mathrm{C} & 6.6(2) \\ \mathrm{O} 1 \mathrm{~A}-\mathrm{O} 1 \mathrm{~A}-\mathrm{C} 1 \mathrm{~A}-\mathrm{C} 7 \mathrm{~A} & 0.00(3) \\ \mathrm{O} 1 \mathrm{~A}-\mathrm{O} 1 \mathrm{~A}-\mathrm{C} 1 \mathrm{~A}-\mathrm{C} 2 \mathrm{~A} & 0.00(2) \\ \mathrm{C} 8 \mathrm{~A}-\mathrm{N} 1 \mathrm{~A}-\mathrm{C} 2 \mathrm{~A}-\mathrm{C} 3 \mathrm{~A} & -0.8(2) \\ \mathrm{C} 8 \mathrm{~A}-\mathrm{N} 1 \mathrm{~A}-\mathrm{C} 2 \mathrm{~A}-\mathrm{C} 1 \mathrm{~A} & 179.80(13) \\ \mathrm{O} 1 \mathrm{~A}-\mathrm{C} 1 \mathrm{~A}-\mathrm{C} 2 \mathrm{~A}-\mathrm{N} 1 \mathrm{~A} & 1.88(17) \\ \mathrm{O} 1 \mathrm{~A}-\mathrm{C} 1 \mathrm{~A}-\mathrm{C} 2 \mathrm{~A}-\mathrm{N} 1 \mathrm{~A} & 1.88(17) \\ \mathrm{C} 7 \mathrm{~A}-\mathrm{C} 1 \mathrm{~A}-\mathrm{C} 2 \mathrm{~A}-\mathrm{N} 1 \mathrm{~A} & -177.78(13) \\ \mathrm{O} 1 \mathrm{~A}-\mathrm{C} 1 \mathrm{~A}-\mathrm{C} 2 \mathrm{~A}-\mathrm{C} 3 \mathrm{~A} & -177.44(13) \\ \mathrm{O} 1 \mathrm{~A}-\mathrm{C} 1 \mathrm{~A}-\mathrm{C} 2 \mathrm{~A}-\mathrm{C} 3 \mathrm{~A} & -177.44(13) \\ \mathrm{C} 7 \mathrm{~A}-\mathrm{C} 1 \mathrm{~A}-\mathrm{C} 2 \mathrm{~A}-\mathrm{C} 3 \mathrm{~A} & 2.9(2) \\ \mathrm{N} 1 \mathrm{~A}-\mathrm{C} 2 \mathrm{~A}-\mathrm{C} 3 \mathrm{~A}-\mathrm{C} 4 \mathrm{~A} & 176.30(16) \\ \mathrm{C} 1 \mathrm{~A}-\mathrm{C} 2 \mathrm{~A}-\mathrm{C} 3 \mathrm{~A}-\mathrm{C} 4 \mathrm{~A} & -4.4(3) \\ \mathrm{C} 2 \mathrm{~A}-\mathrm{C} 3 \mathrm{~A}-\mathrm{C} 4 \mathrm{~A}-\mathrm{C} 5 \mathrm{~A} & 1.6(3) \\ \mathrm{C} 3 \mathrm{~A}-\mathrm{C} 4 \mathrm{~A}-\mathrm{C} 5 \mathrm{~A}-\mathrm{C} 6 \mathrm{~A} & 1.5(3) \\ \mathrm{C} 4 \mathrm{~A}-\mathrm{C} 5 \mathrm{~A}-\mathrm{C} 6 \mathrm{~A}-\mathrm{C} 7 \mathrm{~A} & -0.6(3) \\ \mathrm{C} 5 \mathrm{~A}-\mathrm{C} 6 \mathrm{~A}-\mathrm{C} 7 \mathrm{~A}-\mathrm{C} 1 \mathrm{~A} & -1.8(3) \\ \mathrm{O} 1 \mathrm{~A}-\mathrm{C} 1 \mathrm{~A}-\mathrm{C} 7 \mathrm{~A}-\mathrm{C} 6 \mathrm{~A} & -178.88(16) \\ \mathrm{O} 1 \mathrm{~A}-\mathrm{C} 1 \mathrm{~A}-\mathrm{C} 7 \mathrm{~A}-\mathrm{C} 6 \mathrm{~A} & -178.88(16) \\ \mathrm{C} 2 \mathrm{~A}-\mathrm{C} 1 \mathrm{~A}-\mathrm{C} 7 \mathrm{~A}-\mathrm{C} 6 \mathrm{~A} & 0.8(3) \\ & \end{array}$

Hydrogen-bond geometry $\left(\AA,{ }^{\circ}\right)$

\begin{tabular}{lllll}
\hline$D-\mathrm{H} \cdots A$ & $D-\mathrm{H}$ & $\mathrm{H} \cdots A$ & $D \cdots A$ & $D-\mathrm{H} \cdots A$ \\
\hline $\mathrm{N} 1 A-\mathrm{H} N 1 A \cdots \mathrm{O} 1 A$ & $0.884(17)$ & $2.099(16)$ & $2.5453(16)$ & $110.3(13)$ \\
$\mathrm{N} 1 A-\mathrm{H} 1 A \cdots \mathrm{O} 1 B$ & $0.884(17)$ & $2.248(17)$ & $2.9375(17)$ & $134.6(14)$ \\
$\mathrm{N} 1 B-\mathrm{H} N 1 B \cdots \mathrm{O} 1 B$ & $0.893(15)$ & $2.085(15)$ & $2.5513(16)$ & $111.5(12)$ \\
$\mathrm{N} 1 B-\mathrm{H} N 1 B \cdots \mathrm{O} 1 C$ & $0.893(15)$ & $2.385(15)$ & $3.1566(18)$ & $144.7(13)$ \\
$\mathrm{N} 1 C-\mathrm{H} N 1 C \cdots \mathrm{O} 1 C$ & $0.890(16)$ & $2.130(15)$ & $2.5775(16)$ & $110.3(12)$ \\
$\mathrm{N} 1 C-\mathrm{H} N 1 C \cdots \mathrm{O} 1 A^{\mathrm{i}}$ & $0.890(16)$ & $2.313(16)$ & $2.9759(17)$ & $131.3(13)$ \\
$\mathrm{C} 5 C-\mathrm{H} 5 C \cdots \mathrm{O} 1 B^{\mathrm{ii}}$ & 0.95 & 2.42 & $3.2914(19)$ & 153 \\
$\mathrm{C} 7 B-\mathrm{H} 7 B \cdots \mathrm{O} 1 A$ & 0.95 & 2.42 & $3.3446(19)$ & 165 \\
$\mathrm{C} 8 C-\mathrm{H} 8 C 2 \cdots \mathrm{O} 1 A^{\mathrm{i}}$ & 0.98 & 2.56 & $3.178(2)$ & 121 \\
\hline
\end{tabular}

Symmetry codes: (i) $x,-y+3 / 2, z-1 / 2$; (ii) $x, y-1, z$. 